\title{
ESTIMATIVA TEÓRICA DE APROVEITAMENTO ENERGÉTICO A PARTIR DE BIODIGESTOR NO MUNICÍPIO DE SOUZA-PB
}

\section{THEORETICALESTIMATEOFENERGYUSEBIODIGESTORPARTNERINTHE MUNICIPALITYOFSOUZA-PB}

\author{
VANESSA ROSALES BEZERRA, M.SC. | UEPB \\ LUIS REYES ROSALES MONTERO, Dr. | UFCG \\ VALDERI DUARTE LEITE, Dr. |UEPB \\ ADRIANO OLIVEIRA DA SILVA | UEPB \\ YOHANNA JAMILA VILAR DE BRITO, M.SC. | UEPB \\ CARLOS ANTÔNIO PEREIRA DE LIMA, Dr. |UEPB
}

\begin{abstract}
RESUMO
A falta de tratamento e deposição incorreta de resíduos orgânicos, advindos da produção agropecuária apresenta impactos negativos ao meio ambiente, devido ao seu alto grau poluidor, causam degradação dos recursos hídricos e do solo. Grande parte do beneficiamento e fabricação de produtos na atividade agropecuária depende intrinsicamente da energia elétrica para produção, sendo de fundamental importância para tal atividade. O objetiva-se estimar o potencial de geração de energia elétrica, a partir do aproveitamento energético do gás metano, advindo de resíduos de agropecuária, de um biodigestor localizando na zona rural, como também, a avaliação da viabilidade econômica do sistema. A estimativa de produção de biogás encontrada foi favorável ao uso de biodigestor para geração de energia, salienta-se a importância do dimensionamento e manutenção de maneira correta. Posto isso, o aproveitamento de resíduos agropecuários traz benefícios ambientais como tratamento de resíduos orgânicos e redução dos gases de efeito estufa. Conclui-se que a geração de eletricidade nas propriedades rurais agrega valor financeiro e propicia insumos energéticos renováveis para a produção agropecuária.
\end{abstract}

PALAVRAS-CHAVE: Biogás, biodigestores, eletricidade

\begin{abstract}
The lack of treatment and incorrect deposition of organic waste, resulting from agricultural production has negative impacts on the environment, due to its high degree of pollution, causing degradation of water and soil resources. Much of the processing and manufacturing of products in the agricultural activity depends intrinsically on electric energy for production, being of fundamental importance for such activity. The objective is to estimate the potential of electric power generation, based on the energetic use of methane gas, resulting from agricultural residues, from a biodigester located in the rural area, as well as the evaluation of the economic viability of the system. The biogas production estimate found was favorable to the use of a biodigester for power generation, emphasizing the importance of correctly sizing and maintaining it. That said, the use of agricultural waste brings environmental benefits such as treatment of organic waste and reduction of greenhouse gases. It is concluded that the generation of electricity in rural properties adds financial value and provides renewable energy inputs for agricultural production.
\end{abstract}

KEY WORDS: Biogas, biodigestors, electricity 


\section{INTRODUÇÃO}

O agronegócio é uma atividade econômica em crescimento no Brasil e no mundo atualmente, esse setor abastece com matéria prima grande parte das industrias no mercado mundial, e trabalha com bens de consumo essenciais para o ser humano. Dentre várias práticas na agroindústria pode-se citar a criação de animais em confinamento, este seguimento da economia rural vem ganhando espaço e adaptando-se as novas tendências do avanço da tecnologia na agropecuária.

O Brasil tem potencial considerável de geração de eletricidade a partir do biogás obtido pela digestão anaeróbia de resíduos sólidos orgânicos, que poderia suprir a demanda de energia em locais com altas taxas de produção do mesmo (SALOMON; LORA, 2005).

Segundo Secretaria de Planejamento do estado da Paraíba (2016), dentre as prioridades do Estado da Paraíba inerente a produção de energia, está o mapeamento e diagnóstico das potencialidades energéticas, principalmente energia solar, biomassa e energia eólica, haja vista a necessidade de colaboração na Paraíba referente ao desenvolvimento energético sustentável.

No Brasil, se intensificou o interesse pelo biogás entre as décadas de 70 e 80 , principalmente por parte dos suinocultores. Algumas empresas e programas governamentais induziram e subsidiaram a implantação de muitos biodigestores nas propriedades rurais, com o foco principal na geração de energia (KUNZ, 2010).

Segundo Brasil (2015), o Biogás corresponde a apenas $0,06 \%$ da potência instalada, menos que outras tecnologias renováveis que possuem maiores limitações quanto à instalação e à operação.

No passado, o setor econômico que utilizava o biogás restringia-se apenas para zona rural, em pequenos produtores, mas, atualmente é utilizado um sistema de produção industrial desenvolvido e complexo.

A tecnologia de produção do biogás ainda é incipiente no Brasil. Durante um longo período de 40 anos (1970 a 2010) não teve a importância adequada, sendo considerado um subproduto, sem valor econômico. (BLEY, 2015)

Confinamento é o sistema de criação em que lotes de animais são encerrados em piquetes ou currais com área restrita, e onde os alimentos e água necessários são fornecidos em cochos. É mais propriamente utilizado para a terminação de bovinos, que é a fase da produção que imediatamente antecede o abate do animal, ou seja, envolve o acabamento da carcaça que será comercializada (TOAZZA, DAL MAGRO e SILVA, 2016).

Segundo Plataforma Itaipu de Energias Renováveis (2008), uma unidade de produção de leitões típica, com 5 mil matrizes suínas produz cerca de $250 \mathrm{~m}^{3}$ de dejetos por dia. Esse efluente contém $25.000 \mathrm{mg} / \mathrm{L}$ de Demanda Bioquímica de Oxigênio (DBO), ou seja, a carga orgânica de atividades agropecuária, como por exemplo suinocultura é bastante poluidora, superior a de efluente doméstico, sendo este em média, $600 \mathrm{mg} / \mathrm{L}$.

De acordo com Santos et al. (2017), a produção de biogás na zona rural é a adaptação de modelos de biodigestores que podem ser utilizados de acordo com a necessidade da propriedade e produtor. Do ponto de vista sanitário, o uso de biodigestores para tratamento de resíduos animais, favorece a redução de doenças associadas a falta de saneamento, tais resíduos possuem inúmeros microrganismos patogênicos.

Um dos principais problemas na parte de confinamento de animais é o destino dos dejetos produzidos por estes, além de poluir o meio ambiente com a liberação de poluentes atmosféricos como o gás metano( $\mathrm{CH} 4)$, os dejetos dos animais também propiciam problemas relacionados a saneamento, contaminações de microrganismos que podem ser prejudiciais tanto para os seres humanos como para os próprios animais.

Uma solução alternativa para a realidade da atividade pecuária na zona rural é a implantação de biodigestores nas regiões que possuem criação animal em confinamento. Além de dar um adequado destino para os dejetos, os biodigestores também podem produzir biogás, que alimentados a motores elétricos ciclo otto produzem eletricidade.

No final do século XIX, o biogás passou a ser coletado em estações de tratamento de efluentes na Inglaterra e, na década de 40, começou a ser aproveitado a partir de esterco de animais em plantas de geração de energia na Índia. Desde então, o processo anaeróbio tem evoluído e se expandido para o tratamento de resíduos agrícolas e industriais (PAIXÃO,2018).

O objetivo deste trabalho é estimar o potencial de geração de energia elétrica, a partir do aproveitamento energético do gás metano, advindo de resíduos de agropecuária, de um biodigestor localizando na zona rural, como também, a avaliação da viabilidade econômica do sistema.

\section{METODOLOGIA}

Sousa é um município brasileiro localizado no interior do estado da Paraíba, distante 438 quilômetros a oeste de João Pessoa, capital estadual. Pertence à Região Geográfica Intermediária de Sousa-Cajazeiras e à Região Geográfica Imediata de Sousa. Ocupa uma área de $738,547 \mathrm{~km}^{2}$, dos quais $3,0220 \mathrm{~km}^{2}$ estão em perímetro urbano. Sua população, estimada é 69.554 habitantes, sendo o sexto mais populoso do estado, o primeiro de sua microrregião (IBGE, 2017). 
A temperatura média é uniforme durante o ano, aproximadamente $28.4{ }^{\circ} \mathrm{C}$, favorecendo o investimento de tecnologia de biodigestão anaeróbia, pois apresenta temperaturas altas durante a maior parte do ano, sem necessidade de aquecimento do biodigestor.

O exemplo de gerador de eletricidade a gás , conforme a Figura 1 ,possui as seguintes especificações: Motor 18,0 cv; Potência máxima 9,5 KVA; Potência nominal 8,0 KVA; Rotação 3600 rpm; tensão de saída 110 V / 220 V (bivolt); Sistema de partida elétrica, apresenta o sistema de filtro integrado que permite a conexão do motor diretamente na lona do biodigestor, concluíram que o consumo $4,0 \mathrm{~m}^{3}$ de biogás por hora de trabalho; 3,8 Litros de álcool por hora de trabalho. (ALVARENGA,2016).

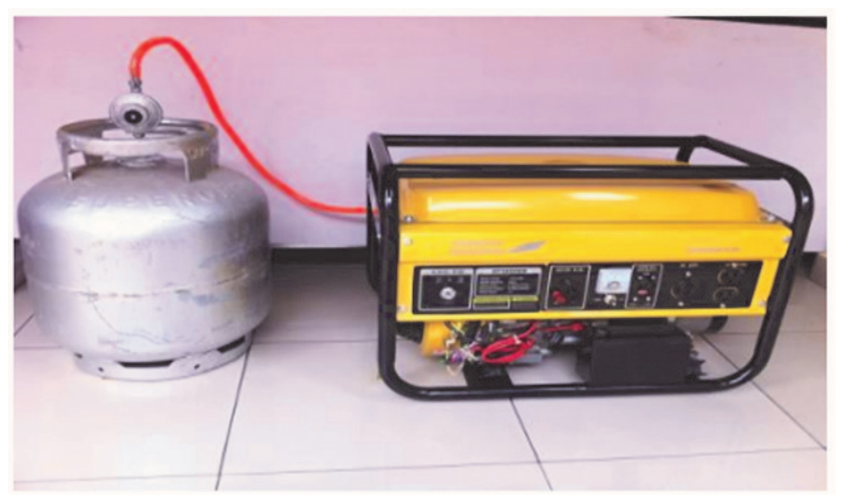

Figura 1 - Gerador de eletricidade a gás

Fonte: Alvarenga, (2015)

Normalmente, o biogás é composto por $60 \%$ de metano, 35\% de dióxido de carbono e 5\% de uma mistura de hidrogênio, nitrogênio, amônia, ácido sulfídrico, monóxido de carbono, aminas e oxigênio, mas dependendo das condições da matéria orgânica, pressão e temperatura durante a fermentação, o biogás pode conter de $40 \%$ a 80\% de metano (SCHWINGEL, 2016) Na Figura 2 apresenta as diversas formas de utilização do biogás.

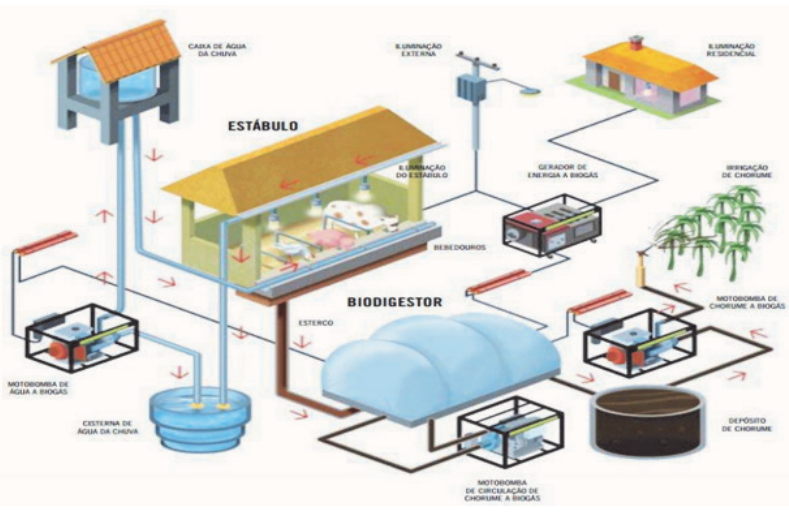

Figura 2 - Utilização do biogás Fonte: ALVARENGA, (2016)
Segundo Stegelin (2010), os benefícios de energia gerada pelo biogás originada de resíduos orgânicos, consistem em economia no custo da energia elétrica comprada, economia com combustível quando o biogás é usado para esse fim.

O dimensionamento do biorreator está projetado para coleta de dejetos de 10 animais suínos e 23 bovinos, localizado em propriedade rural no município de Souza/PB. A implantação do projeto, construção e operação para um biodigestor sertanejo, tem a finalidade principal de avaliar a viabilidade técnica econômica do sistema e o intuito de aproveitamento energético a partir do biogás.

\section{RESULTADOS E DISCUSSÃO}

Foi realizada uma pesquisa sobre projeto de construção de um biodigestor sertanejo. O dimensionamento apresenta os parâmetros operacionais para operação do sistema de forma eficiente, como também a estimativa econômica para projeção de tal reator anaeróbio , considerando características ambientais como temperatura ,e a entrada do substrato no biodigestor, os quais são variáveis importantes para o projeto. As tabelas seguintes são apresentados os dados básicos de projeto.

\begin{tabular}{|l|l|}
\hline Nome do Projeto: & Biodigestor Sertanejo \\
\hline Local: & Souza-PB \\
\hline Data: & $22 / 05 / 2019$ \\
\hline
\end{tabular}

Tabela 1 - Pretensão do projeto

Fonte: Autores

\begin{tabular}{|l|l|l|}
\hline Temperatura mínima: & 22 & ${ }^{\circ} \mathrm{C}$ \\
\hline Temperatura média: & 30 & ${ }^{\circ} \mathrm{C}$ \\
\hline Temperatura máxima: & 35 & ${ }^{\circ} \mathrm{C}$ \\
\hline
\end{tabular}

Tabela 2 - Dados ambientais Fonte: Autores

\begin{tabular}{|l|l|l|l|}
\hline $\mathrm{N}^{\circ}$ & Biomassa (animais: 10 Suínos, 23 Bovinos) & $\mathrm{kg} / \mathrm{d}$ & $\mathrm{m}^{3} / \mathrm{d}$ \\
\hline 1 & Excremento & 506 & 0,506 \\
\hline \multicolumn{2}{|l|}{ Total } & 506,0 & 0,506 \\
\hline
\end{tabular}

Tabela 3 - Resumo de substrato para alimentação do biodigestor Fonte: Autores

\begin{tabular}{|l|l|l|}
\hline Unidades requeridas \\
\hline Tipo: & \multicolumn{2}{|l|}{ Sertanejo } \\
\hline Material: & Alvenaria, PVC, madeira, ferro e Fibra de vidro \\
\hline Volume total requerido: & 15.18 & $\mathrm{~m}^{3}$ \\
\hline número de unidades: & 1 & Global \\
\hline Volume de cada unidade: & 15.4 & $\mathrm{~m}^{3}$ \\
\hline
\end{tabular}




\begin{tabular}{|l|l|l|}
\hline Profundidade: & 3.05 & $\mathrm{~m}$ \\
\hline Diâmetro: & 2.6 & $\mathrm{~m}$ \\
\hline Volume do Gasômetro: & 5.00 & $\mathrm{~m}$ \\
\hline
\end{tabular}

Tabela 4 - Biodigestor

Fonte: Autores

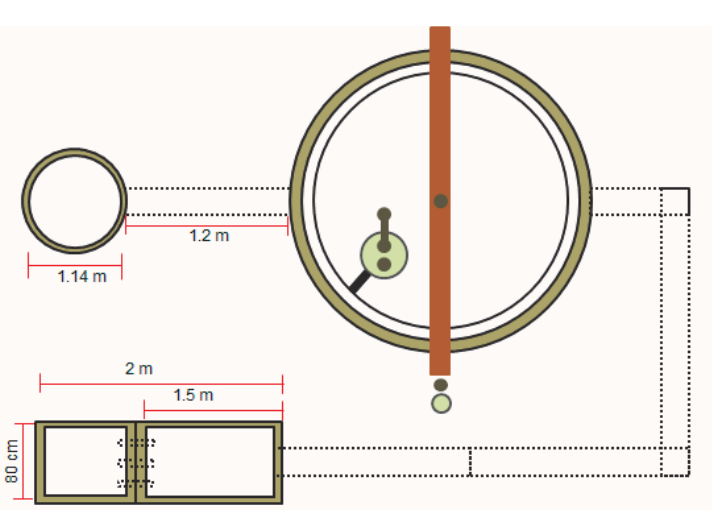

Figura 3 - Modelo de dimensionamento do biodigestor Fonte: Autores

\begin{tabular}{|l|l|l|}
\hline Forma: & Cilíndrica \\
\hline Volume: & 0.51 & $\mathrm{~m}^{3}$ \\
\hline Altura: & 0.5 & $\mathrm{~m}$ \\
\hline Diâmetro: & 1.14 & $\mathrm{~m}$ \\
\hline
\end{tabular}

Tabela 5 - Unidade de alimentação

Fonte: Autores

\begin{tabular}{|l|l|l|}
\hline Forma: & \multicolumn{2}{|l|}{ Retangular } \\
\hline Volume: & 0,80 & $\mathrm{~m}^{3}$ \\
\hline Largura: & 0,80 & $\mathrm{~m}$ \\
\hline Comprimento: & 2 & $\mathrm{~m}$ \\
\hline Profundidade: & 0,50 & $\mathrm{~m}$ \\
\hline
\end{tabular}

Tabela 6 - Unidade de Saída

Fonte: Autores

\section{Produção de biogás, energia e equivalências energéticas}

\begin{tabular}{|l|l|l|}
\hline Descrição & $\mathrm{m}^{3}$ (dia) & $\mathrm{m}^{3}$ (ano) \\
\hline Produção de biogás: & 10.5 & 130.83 \\
\hline Produção de metano: & 7.00 & 82.591 \\
\hline
\end{tabular}

Tabela 7 - Produção de biogás e energia

Fonte: Autores

\begin{tabular}{|l|l|l|}
\hline Descrição & (dia) & (ano) \\
\hline Biogás $\left(\mathrm{Nm}^{3}\right)$ & 10.005 & 3651.825 \\
\hline kWh & 14.507 & 5295.146 \\
\hline $\mathrm{GLP}(\mathrm{kg})$ & 4.50225 & 1643.321 \\
\hline $\mathrm{N}^{\circ}$ de Botijões & 0.3463269 & 126.409 \\
\hline
\end{tabular}

Tabela 8 - Equivalências energéticas de biogás Fonte: Autores

\begin{tabular}{|l|l|l|}
\hline Descrição & $($ Kg/dia $)$ & (t/ano) \\
\hline Massa total de Fertilizante orgânico: & 240.53377 & 87.79 \\
\hline
\end{tabular}

Tabela 9 - Produção de Fertilizante orgânico Fonte: Autores

\section{Orçamento}

\begin{tabular}{|c|c|c|c|c|c|c|c|}
\hline  & $\begin{array}{l}\stackrel{+}{\check{c}} \\
\stackrel{0}{\partial} \\
0\end{array}$ & $\begin{array}{l}\text { हू } \\
\frac{\tilde{E}}{\sigma} \\
\frac{\sigma}{0}\end{array}$ & 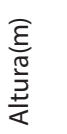 & 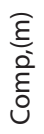 &  &  & 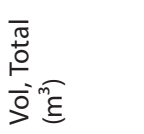 \\
\hline $\begin{array}{l}\text { Tanques de } \\
\text { alimentação }\end{array}$ & 1 & 1.14 & 0.5 & - & - & 0.51 & 0.506 \\
\hline $\begin{array}{l}\text { Biodiges- } \\
\text { tores }\end{array}$ & 1 & 2.6 & 2.9 & - & - & 15.40 & 15.3969456 \\
\hline $\begin{array}{l}\text { Tanque de } \\
\text { descarga }\end{array}$ & 1 & - & 0.50 & 2 & 0.80 & 0.80 & 0.8 \\
\hline \multicolumn{6}{|c|}{ Custo estimado de construção } & (R\$) & $1.869,06$ \\
\hline
\end{tabular}

Tabela 10 - Resumo estrutural

Fonte: Autores

\section{Análise de Custo-Benefício}

\begin{tabular}{|l|l|l|}
\hline & Unid, (R\$/mês) & (R\$/ano) \\
\hline $\begin{array}{l}\text { Filtro de remoção de H2S } \\
\text { (Lã de aço 60g) }\end{array}$ & 2,98 & 35,76 \\
\hline Soma dos custos de manutenção & $\mathbf{( R \$ )}$ & $\mathbf{3 5 , 7 6}$ \\
\hline
\end{tabular}

Tabela 11 - Despesas anuais

Fonte: Autores

\begin{tabular}{|l|l|l|l|}
\hline Energia Calorífera & Botijão/ano & $\mathrm{R} \$ /$ Botijão & $\mathrm{R}$ /ano \\
\hline Economia com botijão de gás & 12 & 70,00 & 5236.46 \\
\hline Fertilizante & $\mathrm{t} / \mathrm{ano}$ & $\mathrm{R} \$ / \mathrm{t}$ & $\mathrm{R} \$ / \mathrm{ano}$ \\
\hline Economia do Fertilizante & 7,61 & 20 & 1755.90 \\
\hline Soma do total de economias anuais & $\mathbf{( R \$ )}$ & $\mathbf{1 . 0 5 5 , 0 5}$ \\
\hline
\end{tabular}

Tabela 12 - Economias anuais

Fonte: Autores

\begin{tabular}{|l|l|}
\hline Resumo de Gastos anuais & R\$/ano \\
\hline Custo de operação e manutenção & 35,76 \\
\hline Soma do custos (R\$) & $\mathbf{3 5 , 7 6}$ \\
\hline
\end{tabular}

\begin{tabular}{|l|l|l|}
\hline \multicolumn{2}{|l|}{ Resumo de Lucros anuais } & R\$/ano \\
\hline Redução de custos com Energia & 5236.46 & 902,84 \\
\hline Fertilizante & 1755.90 & 152,21 \\
\hline Soma do total de rendimento (R\$) & $\mathbf{6 9 9 2 . 3 6}$ & $\mathbf{1 . 0 5 5 , 0 5}$ \\
\hline
\end{tabular}

\begin{tabular}{|l|l|}
\hline Rendimento anual: Lucros - Custos (R\$) & $6.956,60$ \\
\hline Taxa interna de retorno (TIR) & 3.72 \\
\hline
\end{tabular}

Tabela 13 - Resumo de gastos e lucros anuais, rendimento anual e taxa interna de retorno Fonte: Autores 
Ressalta-se que a utilização de biogás para produção de eletricidade na zona rural é pouco difundida, em virtude da falta de informações sobre tecnologias disponíveis e a falta de conhecimento sobre tal assunto.

Zago (2013) avaliou o potencial de produção de eletricidade a partir do biogás, para uma criação de animais suínos, na zona rural da cidade de Santa Catarina e observou que a produção biogás foi cerca de 50 ( $\mathrm{Nm}^{3}$.dia-1),e possuiu a capacidade para geração de 2.160 (kWh.mes-1) . Constatou também, que a propriedades rural pode converter-se em auto suficiente quanto a geração de energia elétrica, com o uso de um sistema com capacidade de 20 KW de potência elétrica, tal pesquisa destacou que o empreendimento é viável economicamente.

A digestão anaeróbia é importante alternativa para o tratamento de resíduos com elevadas concentrações de materiais orgânicos putrescíveis, haja vista incorporar baixos custos operacionais e produzir fonte limpa de energia.

É necessário realizar uma análise de custo/benefício para a utilização dos resíduos sólidos orgânicos da agropecuária para aproveitamento energético, avaliar os custos relacionados a transporte, coleta de resíduos, e o nível de tecnologia para desempenho desta atividade.

Os dejetos suínos sempre foram causadores de grandes fontes de poluição, porém, é necessário destacar que esta poluição pode ser transformada em fontes de riqueza. Uma granja de suínos, além de produzir a carne e outros derivados, pode constituir em excelente produtora de energia, tanto para si, como para terceiros. É importante enfatizar que esse tipo de energia é renovável e a busca pelas tais fontes está crescendo rapidamente em todo o mundo (SILVA; FRANCISCO, 2010).

Grande parte do beneficiamento e fabricação de produtos na atividade agropecuária depende intrinsicamente da energia elétrica para produção, sendo de fundamental importância para tal atividade. Nesta perspectiva, a geração de eletricidade nas propriedades rurais agrega valor financeiro e propicia insumos energéticos renováveis para a produção agropecuária.

Coldebella et al. (2006) analisou a produção de energia elétrica via biogás da bovinocultura em estudo experimental, constatou que o custo de produção de energia elétrica está diretamente associado ao tempo de amortização do investimento e de operação do sistema.

Salienta-se, que as características quanto a tecnologia disponível e viabilidade econômica do aproveitamento do biogás, para produção de energia elétrica no setor agropecuário, deve ser avaliado cada situação de forma diferenciada.
Conforme Santos et al. (2018), entre os principais obstáculos para o aumento do potencial energético derivado do biogás no Brasil é a dificuldade de alcançar a viabilidade econômica, posto que o custo de geração de energia a partir do biogás, de maneira geral é superior à tarifa de venda de energia atualmente no mercado brasileiro.

Portanto, a utilização de biodigestores é viável, porque além de realizar o tratamento de resíduos agropecuários, evitando problemas de contaminação, traz vantagens econômicas ao utilizar corretamente o biogás e os biofertilizantes, que são os subprodutos do processo

\section{CONCLUSÃO}

O uso de biodigestores em fazendas de criação de animais em confinamento apresenta viabilidade técnica-econômica, além de solucionar o problema do destino dos dejetos dos animais, demonstra que é possível o setor rural ser autossustentável no que diz respeito a produção de energia.

Porém, a maior parte dos rebanhos, corresponde à criação extensiva, posto isso, estas estimativas devem ser analisadas de formas distintas, haja vista particularidades de cada criação. Neste contexto, quando a criação de animais ocorre em confinamento, a viabilidade técnica-econômica é mais expressiva.

Grande parte do beneficiamento e fabricação de produtos na atividade agropecuária depende intrinsicamente da energia elétrica para produção, sendo de fundamental importância para tal atividade. Nesta perspectiva, a geração de eletricidade nas propriedades rurais agrega valor financeiro e propicia insumos energéticos renováveis para a produção agropecuária.

Diversas barreiras dificultam a efetiva inserção do biogás na matriz energética brasileira, como a falta de interesse e conhecimento dos gestores públicos para utilização de tal tecnologia renovável.

\section{REFERÊNCIAS}

ALVARENGA,(2006) Frederico de Oliveira Junior. Manual de construção do biodigestorrural.,http:// www1.pucminas.br/imagedb/documento/DOC_ DSC_NOME_ARQUI20140917140023.pdf.

COLDEBELLA, Anderson et al. Viabilidade da cogeração de energia elétrica com biogás da bonivocultura de leite. Proceedings of the 6 . Encontro de Energia no Meio Rural, 2006

BLEY , Júnior C. Biogás: a energia invisível. 2a edição revisada e ampliada. São Paulo: CIBiogás, 2015.

KUNZ, A. Curso de Atualização em Energias do Biogás - EaD. MÓDULO II - Linhas de Base para a Produção 
e Conversão de Energia do Biogás, 2010. Parque Tecnológico de Itaipu (PTI).

MANSO,(2016) Kennia de Jesus. Confinamento de bovinos: Estudo do gerenciamento dos resíduos. Universidade católica de Goiás.

MME, Caracterização Energética da Biomassa Vegetal. BARRETO, Eduardo José Fagundes (Coord.). Combustão e Gaseificação da Biomassa Sólida: Soluções Energéticas para a Amazônia. Brasília: Ministério de Minas e Energia, p.52-63, 2008.

SANTOS, Ivan Felipe Silva et al. Assessment of potential biogas production from multiple organic wastes in Brazil: Impact on energy generation, use, and emissions abatement. Resources, Conservation and Recycling, v. 131, p. 54-63, 2018.

SANTOS, Diego Felipe; GUIMARÃES, Willian Franklin Ferreira; GONÇALVES, Claudio Ubiratan. Biodigestores como alternativa à sustentabilidade ambiental no campo brasileiro: um balanço bibliográfico acerca dos modelos Indiano, Chinês e Batelada. Revista Ciência Agrícola, v. 15, p. 35-39, 2017.

SALOMON, K. R. e LORA, E. E. S. Estimate of The Electric Energy Generating Potential for Different Sources of Biogas in Brazil. Biomass and Bioenergy, v. 33, p. 110111-7, 2009.

STEGELIN, Forrest et al. Oportunidades econômicas, de marketing, ambientais e financeiras sustentáveis para sistemas de recuperação de biogás. In: Pesquisa Agronegocial WERA-72 Enfatizando o Encontro de Competitividade e Rentabilidade, Santa Clara . 2010.

SILVA, Welligton R. et al. Digestão Anaeróbia de Resíduos Vegetais com Baixa Concentração. Gaia Scientia, 2013.

SCHWINGEL, Alice Watte et al. Desempenho da co-digestão anaeróbia de dejetos suínos com inclusão de glicerina bruta. Revista Ciência Agronômica, v. 47, n. 4, p. 778-783, 2016.

OLIVEIRA, P. A. V. de. Produção e aproveitamento do biogás.In: OLIVEIRA, P. A. V. de. Tecnologias para o manejo de resíduos na produção de suínos: manual de boas práticas. Concórdia:Embrapa Suínos e Aves, 2015. Cap. 4, p.43-55.

PARAÍBA, Secretaria planejamento da Paraíba,2016. energias renováveis. Disponível em: paraiba.pb.gov. br/planejamento-e-gestao/ Acesso em:12 jan. 2018.

PAIXÃO, Mateus Vinicius Faria et al. Biogás como fonte energética. Revista Pesquisa e Ação, v. 4, n. 1, p. 129145, 2018.

MATTOS, Luis Cláudio. Manual do biodigestor sertanejo. Recife, 2011.

TOAZZA, Rudinei; DAL MAGRO, Cristian Baú; DA SILVA, Alini. Análise de investimento para ampliação de atividade de confinamento bovino em uma propriedade rural. Revista Brasileira de Contabilidade, n. 221, p. 4051, 2016.

ZAGO, S. Potencialidade de produção de energia através do biogás integrada à melhoria ambiental em propriedades rurais com criação intensiva de animais, na região do meio oeste catarinense.103p. Dissertação de Mestrado, Mestrado em Engenharia Ambiental, Universidade Regional de Blumenau (URB), BlumenauSC, Brasil (2013). 


\section{AUTORES}

ORCID: https://orcid.org/0000-0002-7920-4107

VANESSA ROSALES BEZERRA, M.Sc. | Universidade Estadual da Paraíba | PPGCTA - Doutoranda Engenharia Ambiental| Campina Grande, PB. Brasil | Correspondência para: Rua Juvêncio Arruda, S/N - CEP: 58429-600 - Campus Universitário, Bodocongó - Campina Grande - PB | E-mail: rosalesuepb@gmail.com

ORCID: https://orcid.org/0000-0002-1462-5963

LUIS REYES ROSALES MONTERO, Dr. | Universidade Federal de Campina Grande | DEE - Departamento de Engenharia Elétrica | Campina Grande, PB. Brasil | Correspondência para: Rua Aprígio Veloso, 882 - Bairro Universitário Campina Grande - PB - CEP 58429-900 |E-mail: professorluisreyes@hotmail.com

ORCID: https://orcid.org/ 0000-0001-5861-7407

VALDERI DUARTE LEITE, Dr. | Universidade Estadual da Paraíba | PPGCTA - Programa de Pós Graduação em Ciências e Tecnologia Ambiental | Campina Grande, PB. Brasil | Correspondência para: Rua Juvêncio Arruda, S/N - CEP: 58429-600 - Campus Universitário, Bodocongó - Campina Grande-PB | E-mail: mangabeiraleite@hotmail.com

ORCID: https://orcid.org/0000-0001-9583-8671

ADRIANO OLIVEIRA DA SILVA | Universidade Estadual Da Paraíba | PPGCTA - Programa de Pós Graduação em Ciência e Tecnologia Ambiental | Campina Grande, PB. Brasil | Correspondência para: Rua Juvêncio Arruda, S/N - CEP: 58429-600 - Campus Universitário, Bodocongó - Campina Grande-PB | E-mail: adrianoable222@gmail.com

ORCID: https://orcid.org/0000-0002-8921-3576

YOHHANA JAMILA VILAR DE BRITO , M.Sc. | Universidade Estadual Da Paraíba | PPGCTA - Programa de Pós Graduação em Ciência e Tecnologia Ambiental | Campina Grande, PB. Brasil | Correspondência para: Rua Juvêncio Arruda, S/N - CEP: 58429-600 - Campus Universitário, Bodocongó Campina Grande - PB | E-mail:

ORCID: https://orcid.org/0000-0002-1301-6066

CARLOS ANTÔNIO PEREIRA DE LIMA, Dr. | Universidade Estadual Da Paraíba | PPGCTA - Programa de Pós Graduação em Ciência e Tecnologia Ambiental | Campina Grande, PB. Brasil | Correspondência para: Rua Juvêncio Arruda, S/N - CEP: 58429-600 - Campus Universitário, Bodocongó Campina Grande - PB | E-mail: caplima@uepb.edu.br

\section{COMO CITAR ESTE ARTIGO}

BEZERRA, Vanessa Rosales; MONTERO, Luis Reyes Rolases; LEITE, Valderi Duarte; SILVA, Adriano Oliveira da; BRITO, Yohhana Jamila Vilar de; LIMA, Carlos Antonio Pereira de. Estimativa Teórica de Aproveitamento Energético a partir de Biodigestor no Municipio de Souza-PB. MIX Sustentável, [S.I.], v. 6, n. 4, p. 43-50, ago. 2020. ISSN 24473073. Disponível em:<http://www.nexos.ufsc.br/index.php/mixsustentavel>. Acesso em: dia mês. ano. doi:https://doi. org/10.29183/2447-3073.MIX2020.v6.n4.43-50.

DATA DE ENVIO: $13 / 04 / 2020$

DATA DE ACEITE: $22 / 06 / 2020$ 
